\title{
The Influence of Emotional Factors on Consumption Decision of Infant Formula in China
}

\author{
Ming $X I A^{1, a}$, Jing-Jing GUAN $2, b,{ }^{*}$ \\ ${ }^{1}$ College of Pharmacy, Zhejiang Chinese Medical University, Hangzhou, China \\ ${ }^{2}$ School of Tourism and City Administration, Zhejiang Gongshang University, Hangzhou,China \\ axia1ming2@126.com, bjjguan66@126.com \\ ${ }^{*}$ Corresponding author
}

Keywords: Infant formula, emotional factors, Western culture worship, country of origin effect, halo effect.

\begin{abstract}
In-depth interviews with 48 consumers were conducted to reveal the influence of emotional factors on consumption decision of infant formula, and explore the mechanism. Because of the halo effect, spillover effect and media effect, a negative accumulated mood with homebred infant formula has been generated among Chinese consumers, worship of western culture and region of origin effect has caused a concrete sentiment with imported infant formula at the same time. Eventually, herd mentality of Chinese consumers with imported products has strengthened the advantages of imported infant formula in Chinese market. The reformation of homebred infant formula image can not be improved merely through improvement of quality. To increase the market share of homebred infant formula, reducing Chinese consumer's negative accumulated mood and forming a concrete sentiment with homebred infant formula can be helpful approaches.
\end{abstract}

\section{Introduction}

After the crisis the melamine incident in 2008, the dairy industry in China has had to face new circumstances: Chinese consumers have lost their trust of homebred infant formula. At the same time, they have showed great interests of imported infant formula. Even the rapid increase of imported infant formula price that has been caused by the increase of demand can not stop their desires for purchasing. A general agreement that the quality of imported infant formula is superior to those homemade has been reached among Chinese consumers, although this agreement has insufficient scientific evidences to support with. Understanding the formulation of this emotion tendency and the influence of emotion tendency on consumers' behavior can be meaningful in terms of helping China dairy industry solve their market plight.

In classical research of consumer behavior, the consumer is a rational decision-maker. Buying-decision is a goal-directed process. The consumer makes logical choice among several selective schemes to meet his/her needs. Nevertheless, when facing the choice of infant formula, most Chinese consumers become irrational. Although the average price of homebred infant formula is only half to imported one, and it is even more safety than imported product, and the content of vitamins, minerals, energy-yielding nutrient and other ingredients in homebred infant formula is quite similar to imported product [1], the Chinese consumers insist to purchase imported infant formula. This phenomenon can not be explained using the traditional buying model of knowledge, attitude and practice. Besides knowledge, there is another factor influencing Chinese consumers' purchase decision of infant formula.

The previous studies of Chinese consumers' decision of buying infant formula mostly highlighted the importance of trust [2]. The scholars assumed that the insufficient knowledge decreased consumers' trusts of infant formula, and then the lost of trust negatively influenced their buying intention [3]. However, buying decision is influenced by two kinds of demands, functional demand and emotional demand. Knowledge and trust is more related to functional demand. To explore Chinese consumers' purchase decision of infant formula from another perspective (i.e. emotional demand), this paper investigates the influence of emotional factors on Chinese consumers' purchase 
decision of infant formula, which can obtain a comprehensive picture of consumers' purchase decision of infant formula.

\section{Research Method}

The semi-structure interview approach was used. The interview was conducted on July 2013. The interview questions were showed on Tab 1. Each interview lasted around 20 minutes. All the interviews had been recorded by a digital record, and then transferred into writing text and analyzed.

Three different groups of interviewees were interviewed. The first group is consumers with children who are less than 3 years old and use bottle feeding. Totally 27 consumers in the first group were random selected and interviewed at the reception of The Children's Hospital Zhejiang University School of Medicine. All the interviewees from the first group are among 20-30years old with education background above junior high school. The second group consists of potential consumers. 16 unmarried consumers who are from 20 to 30years old and have above junior high school education background were random approached and interviewed on Mingde road, Hangzhou. The third group has 5 experts from Zhejiang nutrition society. The expertise of experts is food or nutrition science, and all experts have master or doctoral degree.

Table 1 Interview Outlines

\section{No. Content}

1. Usually you buy homebred or imported infant formula and which brand?

2. Do you trust homebred or imported infant formula more? Please state your reasons.

3. Have you consult your relatives and friends before you make purchase decisions? If it is yes, which brand they recommended?

4. What factors have been considered, when you is making buying decision of infant formula?

5. Could you exemplify any negative news of domestic or overseas infant formula? Are those incidents influence your buying decision of infant formula?

6. Even you are aware of that there are some safety issues of overseas infant formula, have you insist on buying imported infant formula? If the answer is positive, what your reasons are?

\section{Research Findings}

\section{Buying-decision of Infant Formula}

Regarding respondents' general buying-decision of infant formula, most respondents (32 of 43 interviewees) indicated that they would like to buy imported infant formula. $18.6 \%$ respondents said that they prefer domestic products. For respondents without clear opinion, they indicated that they would chose imported products, if they have to make purchase decisions, because without more information about product quality, overseas infant formula is more reliable.

When the reasons of buying imported infant formula were asked, the reasons that gave by the respondents who prefer imported infant formula were quite different.

$36.8 \%$ respondents believed that the supervision of production of imported infant is stricter. $17.4 \%$ respondents considered that the production technologies of imported infant formula are more advanced and ingredients of imported products are more scientific. 14.2\% respondents highlighted the sophisticated after-sale service, product recall system and emergency response system of imported infant formula.

Yet, 31.6\% respondents did not have clear reasons to support their buying decisions, and only felt that the imported infant formula is better. Also except experts, none of respondents were able to explain the specific differences of technology, ingredient, supervision and after-sale service between imported and domestic products. Therefore, the viewpoints of imported infant formula having better quality are not formed based on specific and detail data. These viewpoints already became intrinsic 
cognitions. No matter buying imported infant formula or refusing to buy domestic products, the decision making relies on respondents' intrinsic cognitions that imported products are better without specific reasons.

\section{The Influence of Knowledge on Respondents' Buying Decision}

According to respondents, knowledge of infant formula has insignificant influence on their buying decision. Some respondents who already have children indicated that they received a specific class about the ingredient and safety of infant formula at community hospital who has been authorized to teach the knowledge of infant formula to new parents. However, even they had such class, they still prefer imported infant formula. In other words, the knowledge of infant formula has insignificant influence on respondents.

More evidence supporting above statement was from five experts. Even five experts who have sufficient knowledge of infant formula have different viewpoints. Three of them agreed that the quality of imported and homebred infant formula has slight difference, and consumers could purchase infant formula based on their economic condition and preferences. Still there are two experts, one insists imported products are better, and another one considers domestic products are better. Therefore, knowledge of infant formula can not explain the buying decisions of consumers, even experts with great knowledge make different decisions.

\section{The Influence of Relatives and Friends on Respondents' Buying Decision}

Most respondents (19 of 27 respondents) who have been bottle feeding their children admitted that the choices of infant formula were influenced by their friends or relatives. They consulted their friends or relatives' opinions before they made purchase decisions [4]. Some respondents explained that as new parents and who might only have one child in lifetime, they lack of experience and particularly care about the health of their children, therefore they regarded relatives and friends as main and reliable channel of information. Since $63 \%$ recommendations from relatives or friends were usage of imported products, consequently among respondents who take advices from relatives and friends, $77.8 \%$ of them used imported infant formula. Interestingly, some respondents even can not recall the brand or original country of infant formula used, and only remembered that the specific infant formula was recommended by their relatives and friends.

Compared to advices from relatives and friends, respondents indicated that they had less trusts of advices from experts and advertisements. 56.5\% respondents from group one and group two showed their mistrusts of recommendation from experts and advertisements. They explained that experts and advertisements represented the interests of domestic dairy industry, and may not tell truths.

\section{The Influence of Negative Reports on Respondents' Buying Decision}

According to responds of interviews, respondents heard negative reports of both imported and homebred infant formula. However, a few of them can clearly exemplify the unsafe issues of imported infant formula. The negative reports of imported infant formula had slight influences on their purchase decisions. Regarding the homebred products, most respondents not only heard the negative reports, but also can exemplify the unsafe issues. Many respondents mentioned the melamine incident of homebred infant formula in 2008, even this incident happened five years ago. As an important incident of Chinese infant formula, this incident called Chinese consumers attention of qualities of Chinese infant formula, and significantly influenced their purchase decisions. Five years later, $2 / 3$ respondents considered this incident directly caused their purchase of imported infant formula. $46.2 \%$ respondents thought that the melamine incident reflected the malfeasance and dishonesty of Chinese dairy industry. The dairy industry of China is questionable. 
Table 2 The influence of negative reports on respondents' decisions

\begin{tabular}{|l|l|l|l|l|l|l|}
\hline & \multicolumn{2}{|l|}{$\begin{array}{l}\text { Awareness of negative } \\
\text { reports of infant formula }\end{array}$} & \multicolumn{2}{l|}{$\begin{array}{l}\text { Exemplifying } \\
\text { the negative reports }\end{array}$} & \multicolumn{2}{|l|}{$\begin{array}{l}\text { Will negative reports } \\
\text { affect buying decisions }\end{array}$} \\
\cline { 2 - 7 } & Yes & No & Yes & No & Yes & No \\
\hline $\begin{array}{l}\text { Homebred } \\
\text { Infant } \\
\text { formula }\end{array}$ & 96.3 & 3.7 & 77.8 & 22.2 & 76.9 & 23.1 \\
\hline $\begin{array}{l}\text { Imported } \\
\text { Infant } \\
\text { formula }\end{array}$ & 85.2 & 14.8 & 37.0 & 63.0 & 7.4 & 92.6 \\
\hline
\end{tabular}

\section{Discussion}

The findings retrieved from the interviews shows that knowledge of infant formula has slight relationship with consumers' buying decisions, which contradict to statements of many previous studies. Also the respondents indicated that they prefer imported infant formula without specific reasons. These findings show that purchase decisions of infant formula is not a rational buying decision. As an irrational buying decision, emotional factor plays a key role in the decision making.

\section{The Influence of Emotional Factor on Respondents' Buying Decision}

Emotion can be divided into negative emotion and positive emotion [5]. In the context of infant formula purchase, the negative emotion and positive emotion influence consumers' buying-decisions simultaneously.

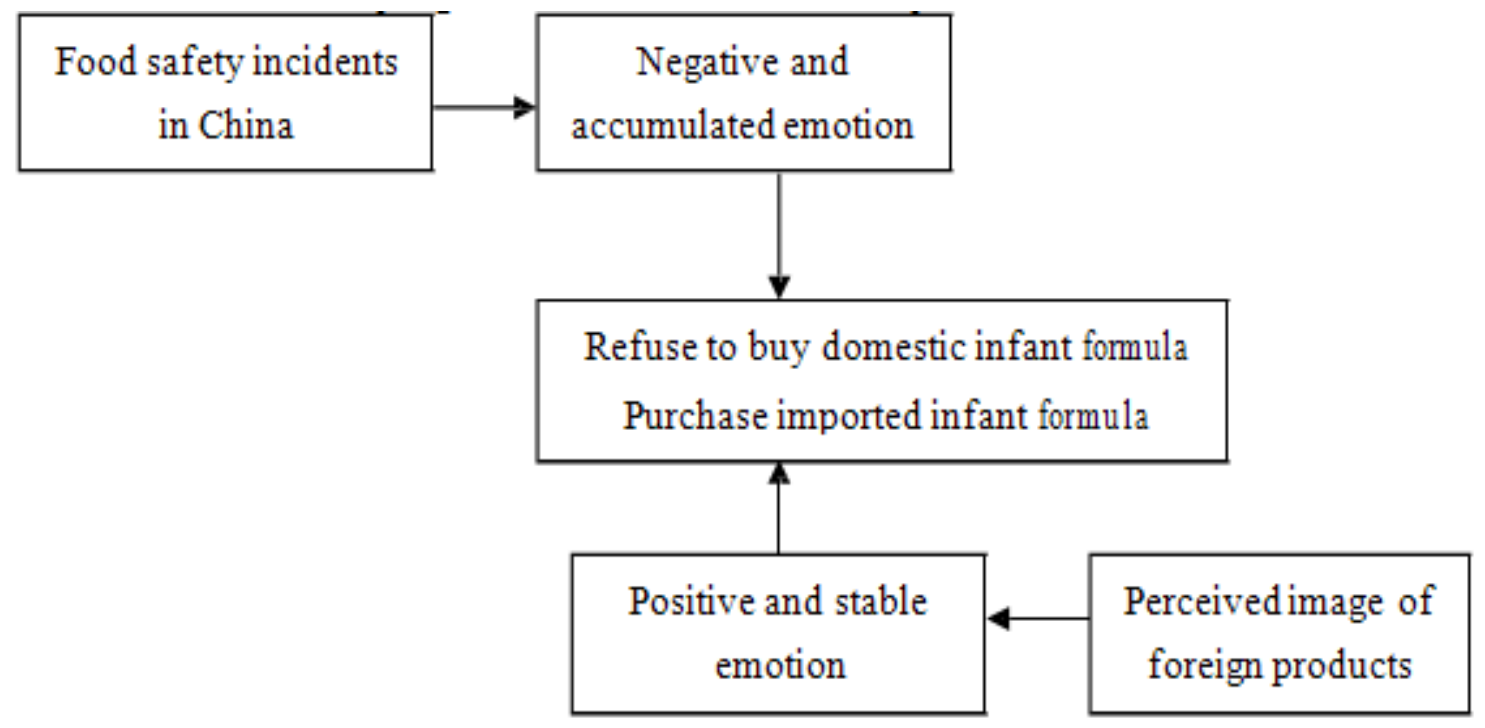

Fig 1 Theoretical model

The melamine incident happened in China in 2008 have significant impact on Chinese consumers' trusts of domestic infant formula, which can be seen from the results of the interviews. The incident aroused consumers' negative emotion towards domestic infant formula. Meanwhile from then on, the food safety incidents occurred constantly in China. These food safety incidents had halo effects and caused the accumulation of negative emotion towards domestic infant formula. Gradually, consumers have psychological distances from Chinese infant formula and do not willing to buy it.

On the other hand, because of brand effects and western adoration, to Chinese consumers, the 
products produced at western countries have automatically been regarded as high quality. The imported infant formula also has established such positive image among Chinese consumers. Therefore, Chinese consumers have positive emotion with imported infant formula, and this emotion is quite stable. Even consumers realize some negative incidents of imported infant formula, they still believe these incidents are accidental and can be tolerated.

The simultaneous effects of negative and positive emotion cause Chinese consumers prefer imported infant formula without specific and clear reasons.

\section{Conformity Phenomenon}

Besides the emotion factor, relatives or friends have significant influence on consumers' buying decision. This is a kind of conformity phenomenon, namely people conformed to the idea of the group. Consumers choose same brand of infant formula as their relative and friends. The conformity phenomenon is particularly obvious among consumers purchasing imported infant formula [6].

\section{Conclusion and Suggestion}

Because of the emotional factor, Chinese consumers make purchase decisions of imported infant formula without specific reason and non-influenced by knowledge of infant formula. The successive incidents of infant formula and food safety have aroused consumers' negative emotion. The Chinese consumers have become irrational. Also conformity effect influents Chinese consumers' final buying decision. In the other words, consumers' negative emotion has been interplayed.

Currently, Chinese consumers' personal emotion and social environment are in favor of the development of infant formula. For the development of domestic infant formula industry, the positive emotion of consumers has to be established. The establishment of the positive emotion relies on the improvement of the quality of domestic products, and the positive reports from media. As the formation of negative emotion towards domestic infant formula has been a process of accumulation, the formation of positive emotion also can not be accomplished immediately. The domestic infant formula industry needs to show its improvement in a long term, and then the positive emotion of consumer can be established.

\section{References}

[1] Liu Ningyu, Xia Ming, Wang Huiming. et.al., Comparison of the Nutrients in Domestic and Oversea Branded Infant Formulas Milk Powder [J]. Food and Nutrition in China 2013, 19(4): 70-72.

[2] Liu Hua, Chen Yan. Influence factors for consumers buying infant milk powder: Based on the survey data of 167 consumers in Nanjing city [J]. Journal of Hunan Agricultural University(Social Sciences). 2013, 14(1): 22-28

[3] Domenico Consoli. Emotions that influence purchase decisions and their electronic processing[C]. Annales Universitatis Apulensis series Oeconomica. 2009.

[4] Elinor Ostrom. Collective Action and the Evolution of Social Norms [J]. The Journal of Economic Perspectives, 2000(13): 137-158.

[5] Yang Qianxuan. The Analysis on Spillover Effect and After-Marketing Management of the Product-Harm Crisis [D]. Wuhan: HuaZhong university of science and technology, 2011.

[6] Wang Jiawei. Behavioral science [M]. Hangzhou: Zhejiang education publishing house, 1986. 